\title{
Superheavy Nuclei to Hypernuclei: A Tribute to Walter Greiner
}

\author{
Chhanda Samanta ${ }^{1, a}$ \\ ${ }^{1}$ Department of Physics and Astronomy, Virginia Military Institute, Lexington, VA 24450, USA
}

\begin{abstract}
In nuclear physics, superheavy and hypernuclei are two of the most important fields of research. The prediction of islands of superheavy elements $(Z=114, N=184$, 196 and $Z=164, N=318$ ) in late sixties by the Frankfurt school played a key role in extending the periodic table of elements up to atomic number 118. Similarly, the demonstration that nuclear matter can be compressed 510 times of its original volume by nuclear shock waves, produced during heavy ion collision, led to the production of singleand double-lambda hypernuclei, as well as anti-matter nuclei. Recent observation of antihypertriton-comprising an antiproton, an antineutron, and an antilambda hyperon, by the STAR collaboration has now made it possible to envision a 3-dimensional nuclear chart of hypernuclei. My own interest in superheavy and hypernuclei was shaped from my first meeting with Walter Greiner at the International Conference on Atomic and Nuclear clusters held at Santorini, Greece in 1993. I will present a brief summary of these exciting developments, including some of our own work. Professor Greiner's vision, enthusiasm, and encouragement touched many lives and I was one of those privileged ones.
\end{abstract}

\section{Introduction}

Walter Greiner wrote in one of his papers [1], "Of particular importance are the substances themselves, i.e., the particles the elements are made of (baryons, mesons, quarks, gluons), i.e., elementary matter." Obviously, the material world has to exist before life began. Thus, the mother of all fundamental questions is: "What are the basic building blocks of the elementary matter?" Understanding of the material world, therefore, depends upon how well we know the elementary building blocks, such as baryons, mesons, quarks, and gluons. Greiner's life-long quest for this understanding not only defined his own scientific career, but influenced many who were lucky enough to come in contact with him. I am one of those physicists who had the good fortune to meet this eminent scientist in 1993 at a conference in Santorini (Greece). Through my interactions with him afterwards on numerous occasions, I realized the importance of unraveling this fundamental truth, and my research hence has been shaped by his inspiration, guidance and support. Over the years, he encouraged me to work on superheavy nuclei, hypernuclei and finally on the dark matter. In each of these fields a large number of theoretical and experimental investigations have already been carried out. I shall present only a few highlights, including some from our own work.

\footnotetext{
a e-mail: samantac@vmi.edu
} 


\section{Superheavy Nuclei}

The heavier the nucleus is, the larger is its Coulomb repulsive force due to increasing number of protons. This makes the transuranium elements more and more unstable leading to a sea of spontaneous fission. In late sixties S. G. Nilsson et al. [2], Mosel and Greiner [3] predicted that for some superheavy nuclei (SHN) the shell effect will counteract the disruptive Coulomb forces. Hence these nuclei can be formed in the midst of the sea of fission. Strong influence of nuclear shells [4] was predicted to produce some SHN with life-time comparable to the age of the earth. These astounding predictions drew considerable attention of experimentalists to investigate the existence of SHN beyond the valley of stability. Since then several searches for superheavy elements in the natural samples have also taken place [5-8], but none has been found in nature so far.

The existence of long-lived SHN is controlled mainly by spontaneous fission and $\alpha$-decay processes. It was believed [6,9-11] that traditional spherical superheavy nuclei might form an "island of stability" centered around ${ }^{298} 114_{184}$ separated from the "peninsula" of known nuclei by a region of deep instability. Since fission barrier and shell effect play very important role for the existence of long lived superheavy nuclei it is crucial to determine the fission barrier and half-life of fissioning nucleus with a good accuracy. It is well known that very small barrier height against fission can break the nucleus into two fragments immediately after it is formed. The $\alpha$ decay of superheavy nuclei is possible if the shell effect supplies the extra binding energy and increases the barrier height of fission. The $\beta$-stable nuclei having relatively longer half-life for spontaneous fission than that for $\alpha$ decay indicates that dominant decay mode for such SHN might be $\alpha$-decay.

Experimental investigations in finding the SHN around $\mathrm{Z}=107-118$ were pursued mainly at three different places: Gesellschaft fur Schwerionenforschung (GSI) in Darmstadt (Germany), Joint Institute for Nuclear research (JINR) in Dubna (Russia), and RIKEN, Japan. In the beginning of the 1980 's the first observations of the elements with $\mathrm{Z}=107-109$ were made at GSI [12]. In 1994, $\alpha$ decay chains were observed from nucleus ${ }^{269} 110$ [13] and later on, $\alpha$-decay chains from nuclides ${ }^{271} 110,{ }^{272} 111,{ }^{277} 112$ [14-16], ${ }^{283} 112$ [17] were detected at GSI.

While RIKEN claimed discovery of the ${ }^{278} 113$ SHN $[18,19]$, it also reconfirmed the $\alpha$ decay chains from ${ }^{271} 110$ [20], ${ }^{272} 111$ [21] and ${ }^{277} 112$ [22]. Observations of the $\alpha$ decay chains of nuclei ${ }^{294} 118,{ }^{290-293} 116,{ }^{288,287} 115,{ }^{286-289} 114,{ }^{282-284} 113,{ }^{285,283} 112$ [23], ${ }^{278-280} 111,{ }^{273,281} 110$ [24, 25], 274-276 $109,{ }^{275} 108,{ }^{272,270} 107,{ }^{271} 106$ were reported by JINR [26-29].

Most of the heavy nuclei are expected to be deformed due to partial filling of large nuclear shells by outer nucleons. According to the theoretical calculations [30, 31], nucleus ${ }^{270}{ }^{2} s_{162}(\mathrm{Z}=108)$ has the features of "deformed doubly magic" nucleus. Dvorak et al. measured the energy $\left(E_{\alpha}\right)$ of $\alpha$ particle emitted from ${ }^{270} \mathrm{Hs}_{162}$ and used the value of $E_{\alpha}$ to calculate $Q_{\alpha}(9.02 \pm 0.03 \mathrm{MeV})$ for the $\alpha$ decay of ${ }^{270}{ }^{27} s_{162}$ [32]. A phenomenological formula [33] estimated the $\alpha$ decay half-life $\sim 22 s$.

According to microscopic nuclear theory [34], "spherical" shell effects at $Z=114,120,126$ and $\mathrm{N}=184$ can provide the extra stability to some SHN to have long enough lifetime to be observed.

To investigate whether the so-called "stability island" could really exist around the said Z, N values, the $\alpha$-decay half lives along with the spontaneous fission and $\beta$-decay half lives of such nuclei were studied by us [35-40]. The $\alpha$-decay half lives of SHN with Z=102-120 (Fig. 1 and Fig.2) were calculated in a quantum tunneling model with DDM3Y effective nuclear interaction using $Q_{\alpha}$ values from three different mass formulae prescribed by Koura, Uno, Tachibana, Yamada (KUTY) [41], Myers, Swiatecki (MS) [42] and Muntian, Hofmann, Patyk, Sobiczewski (M) [43] . Calculation of spontaneous fission (SF) half lives for the same SHN are carried out using a phenomenological formula and compared with SF half lives predicted by Smolanczuk et al. In the region of Z=106-108 with $\mathrm{N} \sim 160-164$, the $\beta$-stable SHN ${ }_{106}^{268} S g_{162}$ is predicted to have highest $\alpha$-decay half life $\left(T_{\alpha} \sim\right.$ 3.2hrs) using $Q_{\alpha}$ value from M. A few fission-survived long-lived SHN which are either $\beta$-stable 


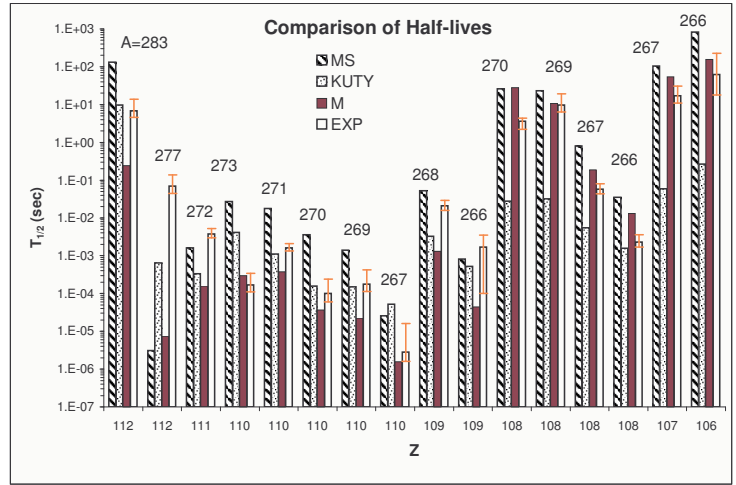

Figure 1. Comparison of half lives for SHN with $\mathrm{Z}=106-112$ [40].

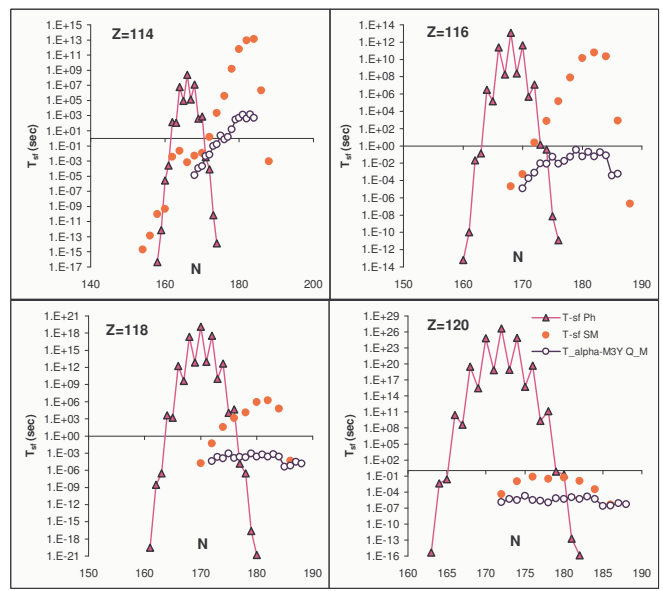

Figure 2. Plots for variation of phenomenological [44, 45] and microscopically [46, 47] calculated fission half lives with neutron numbers. Continuous line with solid circle ( $T_{\alpha} \mathrm{M} 3 \mathrm{Y}$ Q-M) represents $\alpha$ decay half-lives predicted [40] using Q value from Muntian et al [43, 48, 49]. Continuous line with solid triangle (Tsf $\mathrm{Ph}$ ) represents spontaneous fission half-lives predicted by phenomenological calculation. Solid dark circle (Tsf SM) shows the spontaneous fission half-lives predicted by microscopic calculation

or having large $\beta$-decay half lives were predicted to exist near ${ }^{294} 110_{184},{ }^{293} 110_{183},{ }^{296} 112_{184}$ and ${ }^{298} 114_{184}$. These nuclei might decay predominantly through $\alpha$-particle emission.

The heaviest element discovered so far through experiments has 118 protons, and in the year 2016 it has been named, 'Oganesson $(O g)^{\prime}$ '. This superheavy element was first synthesized in 2002 by a 
joint team of Russian and American scientists at the Joint Institute for Nuclear Research (JINR) in Dubna, Russia. ${ }^{294} \mathrm{Og}$ decays into ${ }^{290} \mathrm{Lv}$ by alpha decay. Since there were only three nuclei, the halflife derived from observed lifetimes has a large uncertainty: $0.89_{0.31}^{+1.07} \mathrm{~ms}$ [26]. In a quantum-tunneling model, we had predicted the alpha decay half-life of ${ }^{294} \mathrm{Og}$ to be $0.66_{-0.18}^{0.23} \mathrm{~ms}$ [35]. With the success of discovery of the element with $Z=118$, new searches have started for SHN with $Z=120$. At present no clear signature of this SHN has been identified. Among many other hurdles, extremely small production cross-section is a great hindrance [50] that calls for a huge improvement of the current technology.

\section{Hyperuclei}

The hypernuclear physics is of great importance in many branches of physics. Of particular interest is the understanding of strange particles in baryonic matter, since many questions in heavy-ion physics, particle physics and astrophysics are related to the effect of strangeness (S) in nuclear matter [51]. A hyperon has strange quarks (s) in addition to up (u) and down (d) quarks. The normal matter has proton ( $\mathrm{p}=\mathrm{uud})$ and neutron $(\mathrm{n}=\mathrm{udd})$, and the strange matted has hyperons like Lambda $\left(\Lambda^{o}=u d s\right)$, Sigma-plus $\left(\Sigma^{+}=u u s\right)$, Cascade-minus $\left(\Xi^{-}=d s s\right)$ etc. A hypernucleus contains hyperon(s) in addition to proton and neutrons.

So far about $35 \Lambda$-hypernuclei and 6 doubly-strange $\Lambda \Lambda$-hypernuclei have been produced in the laboratory. Doubly-strange hypernuclei also arise having a $\Xi(S=-2)$ hyperon that has been studied both theoretically [52] and experimentally [53], [54], [55] in a limited number of nuclei. Sigma hyperons exhibit interesting property in its interaction with nuclei. Several studies suggest that due to strongly repulsive $\Sigma$-nucleus potential Sigmas are unbound in nuclei, except for the very special case of nuclei with mass number $\mathrm{A}=4$ [56].

Binding energies of a few hypernuclei have been studied in quark mean Field (QMF) and relativistic mean field (RMF) models[57, 58]. We prescribed a generalized mass formula, applicable to both normal and hyper-nuclei, in which a hypernucleus is considered as a core of a normal nucleus plus hyperon(s). A generalized mass formula for non-strange normal nuclei (with $\mathrm{N}$ number of neutrons and $Z_{c}$ number of protons) and strange hypernuclei (with $n_{Y}$ number of hyperons, each of mass $m_{Y}$, charge $q_{Y}$ and strangeness $S$ ) with total charge $Z=Z_{c}+n_{Y} q_{Y}$, was proposed earlier in a SU(6) symmetry breaking framework [59]. This mass formula is recently optimized with the newly available more accurate data on $\Lambda \Lambda$-hypernuclei summarized in Ref. [51]. It led to a minor modification of one of its parameters from 26.7 to 27.8 in the "strange" part of the formula. The binding energy in this revised formula is given by,

$$
\begin{array}{r}
B(A, Z)=15.777 A-18.34 A^{2 / 3}-0.71 Z(Z-1) / A^{1 / 3}-23.21\left(N-Z_{c}\right)^{2} /\left[\left(1+e^{-A / 17}\right) A\right] \\
+n_{Y}\left[0.0335 m_{Y}-27.8-48.7|S| / A^{2 / 3}\right]+\delta
\end{array}
$$

Where, the pairing term for even $Z_{c}$ - even $\mathrm{N}$ is, $\delta=+12 A^{-1 / 2}\left(1-e^{-A / 30}\right)$, for odd $Z_{c}$ - odd $\mathrm{N}$, $\delta=-12 A^{-1 / 2}\left(1-e^{-A / 30}\right)$, and $\delta=0$ when $N+Z_{c}$ is odd. Here, the mass number $\left(A=N+Z_{c}+n_{Y}\right)$ of the hypernucleus is the sum of number of neutrons $(\mathrm{N})$, protons $\left(Z_{c}\right)$ and number of hyperons $\left(n_{Y}\right)$. The separation energies $\left(S_{Y}\right)$ of a hyperon $(\mathrm{Y})$ from a hypernucleus is given by,

$$
B_{Y}=B(A, Z)_{\text {hyper }}-B\left(A-n_{Y}, Z_{c}\right)_{\text {core }}
$$

The $B_{\Lambda}$ and $B_{\Lambda \Lambda}$ are $\Lambda$ and $\Lambda \Lambda$-binding energies in their respective hypernucleus, ${ }_{\Lambda}^{A} Z$ and ${ }_{\Lambda \Lambda}^{A} Z$. 


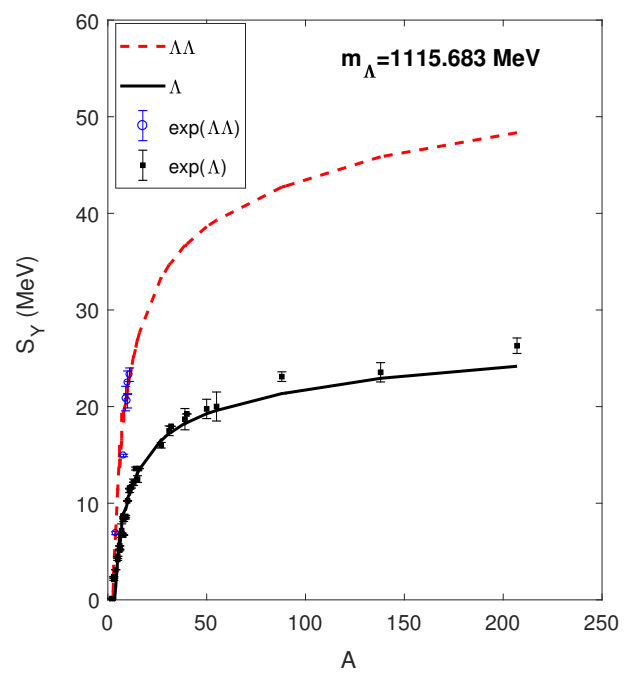

Figure 3. Plot of $\Lambda$ and $\Lambda \Lambda$-separation Energy $\left(S_{Y}\right)$ versus mass Number (A) for single- $\Lambda$ and double- $\Lambda$ hypernuclei, calculated using this mass formula and comparison with the available experimental data.

Fig. 3 shows that the rsults of the mass formula agree with the experimental data $[51,57]$ on the $\Lambda$ and $\Lambda \Lambda$ hypernuclei. We explored the possibility of the existence of bound hypernuclei beyond the normal neutron-drip line and found that the hyperons can help a nucleus to hold more neutrons [60].

The bond energies $\left(\Delta B_{\Lambda \Lambda}\right)$ are the measures of the energy released when the $\Lambda \Lambda$-bond is broken. These energies assist in understanding the nature of the in-medium strength of the $\Lambda \Lambda$-interaction. The $\Lambda \Lambda$-bond energy is defined as,

$$
\Delta B_{\Lambda \Lambda}\left({ }_{\Lambda \Lambda}^{A} Z\right)=B_{\Lambda \Lambda}\left({ }_{\Lambda \Lambda}^{A} Z\right)-2 B_{\Lambda}\left({ }_{\Lambda}^{A-1} Z\right)
$$

In Fig.4, the $\Lambda \Lambda$-Bond energy is plotted against the Mass Number A. The Bond Energies calculated by the generalized mass formula are found to be in good agreement with the experimental data and predictions of Quark Mean Field (QMF) and Relativistic Mean Field (RMF) calculations [57], except at small mass number where the experimental data have large error bars. Also the data point at $A=10$ is deeply negative which could be an extraction error of the data.

Another interesting topic is the observed large charge symmetry breaking in light nuclei[61, 62]. The pair of hypernuclei with the same total number of baryons $(A=N+Z+\Lambda)$, but the neutron $(N)$ and proton $(\mathrm{Z})$ numbers interchanged, are called mirror nuclei. A large difference in binding energies $\left(B_{\Lambda}\right)$ between ${ }_{\Lambda}^{4} \mathrm{H}(\mathrm{N}=2, \mathrm{Z}=1, \Lambda=1)$ and ${ }_{\Lambda}^{4} \mathrm{He}(\mathrm{N}=1, \mathrm{Z}=2, \Lambda=1)$ was observed in the experimental data and it was attributed to charge symmetry breaking (CSB) effect [63]. The CSB is calculated as,

$$
\Delta B_{\Lambda}=B_{\Lambda}\left({ }_{\Lambda}^{N+Z+\Lambda} Z\right)-B_{\Lambda}\left({ }_{\Lambda}^{Z^{\prime}+N+\Lambda} Z^{\prime}\right)
$$

Recently Botta et al. [64] have summarized some of the CSB values that shows that the $\Lambda \mathrm{N}$ interaction is not charge independent. We calculated binding energy differences of several mirror nuclei. The CSB is not predicted by our calculation, since our mass formula gives the Coulomb 


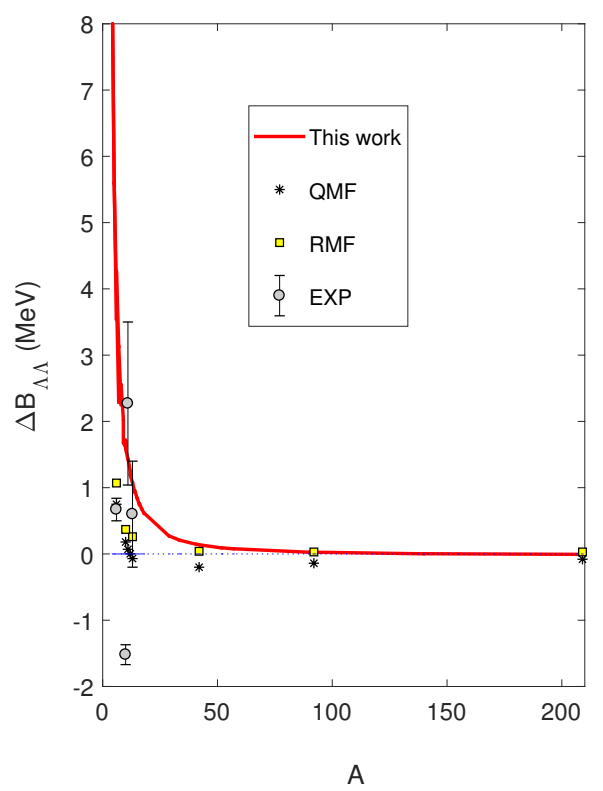

Figure 4. Plot of calculated $\Lambda \Lambda$ bond energies $\left(\Delta B_{\Lambda \Lambda}\right)$ versus the mass number $A$, and comparison with the experimental data, QMF and RMF predictions[57].

energy difference. Nevertheless, this mass formula can be used to extract the Coulomb-corrected CSB from the experimental data. The Coulomb difference is found to have a small A dependence.

In summary this mass formula suggests that some hypernuclei can exist beyond the neutron-drip line of normal nuclei. Finding such neutron-rich hypernuclei experimentally (or, even proving their absence) can shed light on the unknown intricacies of the $\Lambda \mathrm{N}$ interactions in a neutron-rich environment. The $\Lambda \Lambda$-bond energy is found to decrease with increasing neutron number. This information is useful in model calculations for neutron stars. For mirror nuclei this mass formula provides the Coulomb energy differences that show a mild A-dependence. One can extract the Coulomb-corrected CSB effect from the experimental data using this mass formula. More experimental data, especially for neutron-rich light hypernuclei $[65,66]$, are needed and this mass formula can provide a guideline for future experiments on hypernuclei.

\section{Dark matter}

Recent advances in cosmological precision tests consolidate the minimal cosmological standard model, indicating that the universe contains about $4.9 \%$ ordinary matter, $26.8 \%$ dark matter and $68.3 \%$ dark energy. Although being five times more abundant than ordinary matter, the basic properties of dark matter, such as particle mass and interactions are still unknown. Various theoretical models of dark matter exist, ranging from Cold Dark Matter to Warm Dark Matter to Hot Dark Matter and from Symmetric to Asymmetric Dark Matter [67-71].

We considered fermionic Asymmetric Dark Matter (ADM) particles of mass $1 \mathrm{GeV}$ mixed with rotating and non-rotating neutron stars [72]. ADM, like ordinary baryonic matter, is charge asymmetric with only the dark baryon (or generally only the particle) excess remains after the annihilation of 


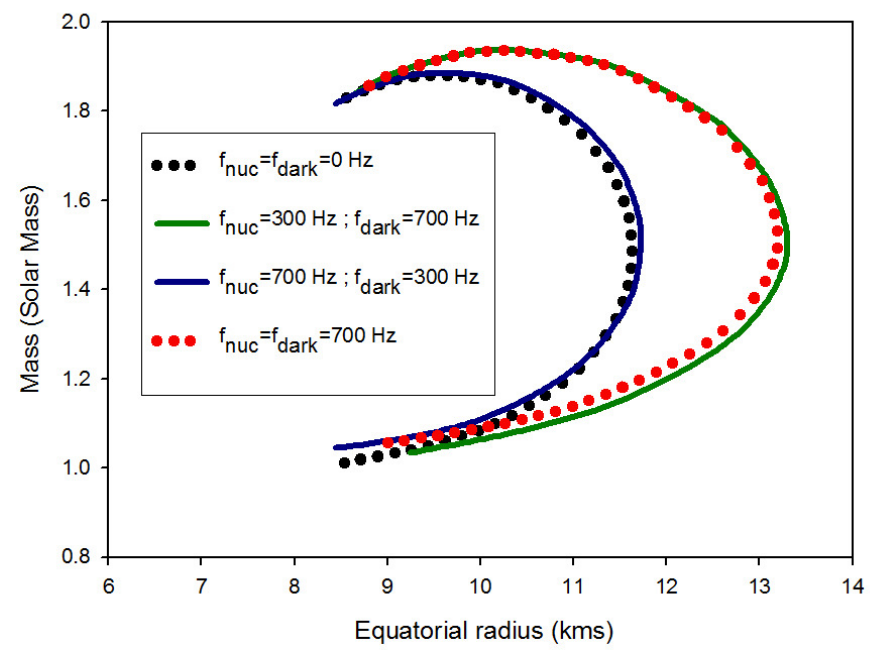

Figure 5. Plots of total mass vs. equatorial radius of static, rigidly rotating and differentially rotating neutron stars mixed with interacting fermionic Asymmetric Dark Matter with fixed nuclear matter central enthalpy $\left(0.24 c^{2}\right)$ and varying dark matter central enthalpies.

most antiparticles after the Big Bang. Hence these ADM particles are non self-annihilating and behaves like ordinary free particles. The gravitational stability and mass-radius relations of static, rigid and differentially rotating neutron stars mixed with fermionic ADM are calculated using the LORENE code [73]. It is important to note that we do not allow any phase transition of the nuclear matter and the interaction between nuclear matter and dark matter is only through gravity.

The nuclear matter Equation of State (EoS) is calculated using the isoscalar and the isovector [74, 75] components of $\mathrm{M} 3 \mathrm{Y}$ interaction along with density dependence. We consider the non-interacting fermionic ADM to be a completely degenerate free Fermi gas of particle mass $m_{\chi}$ at zero temperature. By the Pauli exclusion principle, no quantum state can be occupied by more than one fermion with an identical set of quantum numbers. Thus a noninteracting Fermi gas, unlike a Bose gas, is prohibited from condensing into a Bose-Einstein condensate. The total energy of the Fermi gas at absolute zero is larger than the sum of the single-particle ground states because the Pauli principle implies a degeneracy pressure that keeps fermions separated and moving.

The mass-radius relationship of non-rotating, rigidly rotating and differentially rotating neutron stars admixed with dark matter is calculated using the LORENE code. The nuclear matter and dark matter EoSs are fitted to a polytropic form $P=K \rho^{\gamma}$ where $P$ is the pressure, $\rho$ is the mass density, $K$ the polytropic constant and $\gamma$ the polytropic index for the corresponding fluid. For interacting nuclear matter $\gamma=2.03$ and $K=5.65283 \times 10^{35}$ in C.G.S. units. For interacting dark matter $\gamma=1.97562$ and $K=1.33404 \times 10^{36}$ in C.G.S. units. We take dark matter particle mass to be of $1 \mathrm{GeV}$ and the exchange boson mass $m_{I}=100 \mathrm{MeV}$, typical of strong interaction. First, we keep the dark matter central enthalpy to be $0.24 c^{2}$ (fixed) and vary the nuclear matter central enthalpy for static, rigidly rotating and differentially rotating configurations and next we reverse the roles of nuclear and dark matter. 


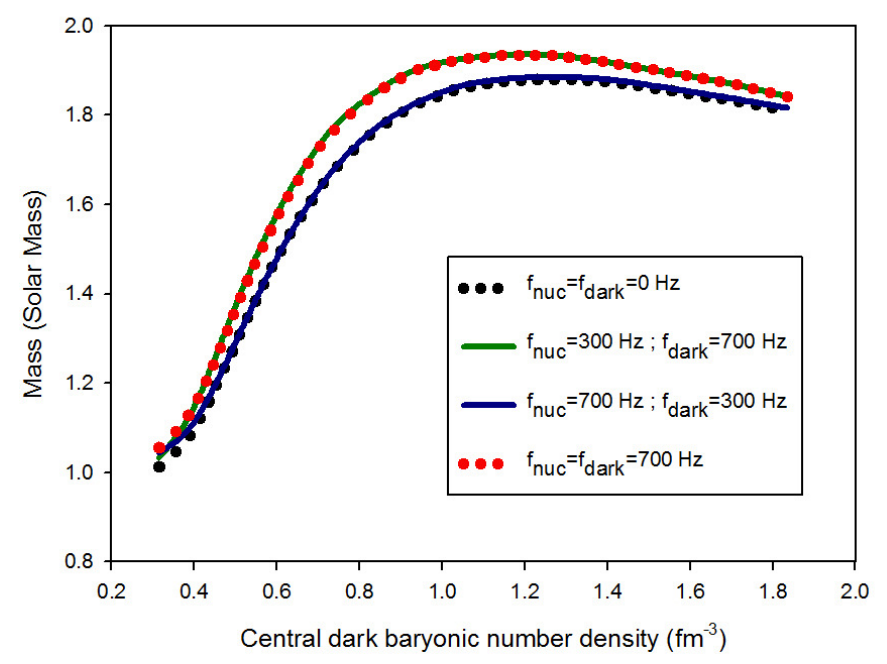

Figure 6. Plots of total mass vs. central dark matter density of static, rigidly rotating and differentially rotating neutron stars mixed with self-interacting fermionic Asymmetric Dark Matter with fixed nuclear matter central enthalpy $\left(0.24 c^{2}\right)$ and varying dark matter central enthalpies.

In Fig.5 the plots of total mass vs. equatorial radius of static, rigidly and differentially rotating neutron stars mixed with fermionic self-interacting dark matter are shown for fixed nuclear matter central enthalpy $\left(0.24 c^{2}\right)$ and varying dark matter central enthalpies. In Fig.6 the corresponding plots of mass vs. central dark baryonic number density are shown.

It is seen that the polytropic indices $\gamma$ for nuclear and self-interacting dark matter EoSs are approximately equal, but the polytropic coefficient $K$ for dark matter is about 2.5 times larger than that of nuclear matter making dark matter EoS stiffer. Consequently, configurations of stars with varying dark matter central enthalpy with fixed nuclear matter central enthalpy are more massive than those obtained for the reverse case.

The maximum mass for non-rotating dark matter stars goes to $3.0279 M_{\odot}$ with a radius of 16.2349 $\mathrm{kms}$ for particle mass $m_{\chi}=1 \mathrm{GeV}$, and that for rotating stars it goes to $3.1460 M_{\odot}$ with a radius of $19.2173 \mathrm{kms}$. However, if one takes $m_{\chi}$ to be $0.5 \mathrm{GeV}$, then the maximum mass goes to $\sim 12.6 M_{\odot}$ using the relation Mass $\propto 1 / m_{\chi}^{2}$ [76], thus mimicking stellar mass black holes. Although, mass of a pure neutron star corresponding to stiff EoS can reach $\sim 2 M_{\odot}$, but accretion of dark matter causes its reduction.

In summary, we took different cases of configurations viz. static, rigid rotation and differential rotation of nuclear matter and dark matter. From the results we conclude that in the rigid and differential rotational cases the maximum mass of these hybrid stars can reach upto $\sim 1.94 M_{\odot}$ with corresponding radii $\sim 10.4 \mathrm{kms}$. The neutron stars admixed with dark matter can lower the neutron star maximum mass. A further interesting study is to explore the effect of dark matter on the nuclear to quark matter phase transition in neutron stars and the corresponding change in the mass limits. We have not studied any such phase transition in the present work but will leave it for future. 


\section{References}

[1] W. Greiner, Jour. Phys: Conference series 337, 012002 (2012).

[2] S. G. Nilsson et al., Nucl. Phys. A 115, 545 (1968).

[3] U. Mosel and W. Greiner, Z. Phys. 217 (1968) 256, 222, 261 (1969).

[4] Z. Patyk, A. Sobiczewski, P. Armbruster, K. H. Schmidt, Nucl. Phys. A 491, 267 (1989).

[5] Yu. A. Muzychka, Phys. Lett. B 28, 539 (1969).

[6] E. O. Fiset and J. R. Nix, Nucl. Phys. A 193, 647 (1972).

[7] V. M. Strutinski and Yu. A. Muzychka, Proc. Int. Conf. on Heavy Ion Physics (JINR, Dubna, 1966) Part-2, 51 (1996).

[8] G. N. Flerov and G. M. Ter-Akopian, Rep. Prog. Phys. 46, 817 (1983).

[9] S.G. Nilssson et al., Nucl. Phys. A115, 545 (1968).

[10] S.G. Nilssson et al., Nucl. Phys. A131, 1 (1969).

[11] J. Randrup, S. E. Larsson, P. Moller, A. Sobiczewski, and A. Lukasiak, Phys. Scr. 10A, 60 (1974).

[12] P. Armbruster, Acta Phys. Pol. B 34, 1825 (2003).

[13] S. Hofmann et al., Z. Phys. A 350, 277 (1995).

[14] S. Hofmann et al., Z. Phys. A 350, 281 (1995).

[15] S. Hofmann et al., Z. Phys. A 354, 229 (1996).

[16] S. Hofmann et al., Rep. Prog. Phys. 61, 639 (1998).

[17] S. Hofmann et al., Euro. Phys. Jour. A32, 251(2007).

[18] K. Morita et al., J. Phys. Soc. Jpn. 73, 2593 (2004).

[19] K. Morita et al., J. Phys. Soc. Jpn. 76, 045001 (2007).

[20] K. Morita et al., Euro. Phys. Jour. A21, 257(2004); T. N. Ginter et al., Phys. Rev. C 67, 064609 (2003).

[21] K. Morita et al., J. Phys. Soc. Jpn. 73,1738 (2004).

[22] K. Morita et al., J. Phys. Soc. Jpn. 76, 043201 (2007).

[23] R. Eichler et al., Nucl. Phys. A 787, 373 (2007).

[24] Yu.A. Lazarev et al., Phys. Rev. C 54, 620 (1996).

[25] Yu.Ts. Oganessian et al., Phys. Rev. Lett. 83, 3154 (1999).

[26] Yu.Ts. Oganessian et al., Phys. Rev. C 74, 044602 (2006); see references therein.

[27] Yu.Ts. Oganessian et al., Phys. Rev. C 69, 021601(R) (2004); Phys. Rev. C 72, 034611 (2005).

[28] Yu.Ts. Oganessian et al., Phys. Rev. C 70, 064609 (2004); Phys. Rev. C 71, 029902(E) (2005).

[29] Yu.Ts. Oganessian et al., Phys. Rev. C 76, 011601 (R) (2007); J. Phys. G 34, R165 (2007).

[30] Z. Patyk and A. Sobiczewski, Nucl. Phys. A 533, 132 (1991).

[31] Z. Patyk and A. Sobiczewski, Phys. Lett. B 256, 207 (1991).

[32] J. Dvorak et al., Phys. Rev. Lett. 97, 242501 (2006).

[33] A. Parkhomenko and A. Sobiczewski, Acta Phys. Pol. B 36, 3095 (2005).

[34] S. Ćwiok, P.-H. Heenen W. Nazarewicz, Nature 433 (2005) 705

[35] P. Roy Chowdhury, C. Samanta and D.N. Basu, Phys. Rev.C 73, 014612 (2006).

[36] C. Samanta, P. Roy Chowdhury and D.N. Basu, Nucl. Phys.A 789, 142 (2007).

[37] P. Roy Chowdhury, D.N. Basu and C. Samanta, Phys. Rev. C 75, 047306 (2007).

[38] C. Samanta, D.N. Basu and P. Roy Chowdhury, J. Phys. Soc. Jpn. 76, 124201 (2007).

[39] P. Roy Chowdhury, C. Samanta and D.N. Basu, Atomic Data and Nuclear Data Tables 94, 781 (2008). 
[40] P. Roy Chowdhury, C. Samanta and D.N. Basu, Phys. Rev.C 77, 044603 (2008) .

[41] H. Koura, M. Uno, T. Tachibana, and M. Yamada, Nucl.Phys. A 674, 47 (2000).

[42] W.D. Myers and W.J. Swiatecki, Nucl. Phys. A 601, 141 (1996).

[43] I. Muntian, S. Hofmann, Z. Patyk and A. Sobiczewski, Acta Phys. Pol. B 34, 2073 (2003).

[44] C. Xu and Z. Ren, Phys. Rev. C 71, 014309 (2005).

[45] Z. Ren and C. Xu, Nucl. Phys. A 759, 64 (2005).

[46] R. Smolanczuk, J. Skalski, and A. Sobiczewski, Phys. Rev. C 52, 1871 (1995).

[47] R. Smolanczuk, Phys. Rev. C 56, 812 (1997).

[48] I. Muntian, Z. Patyk and A. Sobiczewski, Acta Phys. Pol. B 32, 691 (2001).

[49] I. Muntian, Z. Patyk and A. Sobiczewski, Phys. At. Nucl. 66, 1015 (2003).

[50] S. Hofmann et al, The European Physical Journal A 52 (2016) 180

[51] A. Gal, E.V. Hungerford, and D. J. Millener, Rev. Mod. Phys.88, 035004 (2016).

[52] Carl B. Dover and A.Gal 1993 Nucl. Phys. A 560, 559.

[53] H. Bando, T. Motoba and J. Zofka, Int. J. Mod. Phys. A 5, 4021 1990; see references therein.

[54] T. Fukuda et al., Phys. Rev. C 58, 1306 (1998).

[55] P. Khaustov et al. , Phys. Rev. C 61, 054603 (2000).

[56] P.K. Saha et al., Phys. Rev. C 70, 044613 (2004).

[57] J.N. Hu, A. Li, H. Shen, H. Toki, Prog. Theor. Exp. Phys. 013002 (2011).

[58] J.N. Hu, A. Li, H. Shen, Phys. Rev. C 96054304 (2017).

[59] C.Samanta, P.Roy Chowdhury, D.N. Basu,J. Phys.G32, 363 (2006).

[60] C.Samanta, P.Roy Chowdhury, D.N. Basu, J. Phys.G35, 065101 (2006).

[61] P. Achenbach, Few-Body Syst.58, 1 (2017).

[62] A. Gal, Phys. Lett. B744, 352 (2015).

[63] A. R. Bodmer and Q. N. Usmani, Phys. Rev. C 31, 1400 (1985)

[64] E.Botta,T.Bressani, A.Feliciello, Nucl. Phys. A960, 165 (2017).

[65] K. Nakazawa et al, Phys. Procedia 80, 69 (2015).

[66] T. Gogami et al, Phys. Rev C94, 021302 (2016) ; H. Sugimura et al, Phys. Lett. B729, 39 (2014).

[67] Gianfranco Bertone, Dan Hooper and Joseph Silk Physics Reports 405, 279 (2005).

[68] George R. Blumenthal, S. M. Faber, Joel R. Primack and Martin J. Rees Nature 311, 517 (1984).

[69] S. King and A. Merle JCAP 016, 1208 (2012).

[70] C. S. Frenk and S. D. M. White Ann. Phys. 524, 507 (2012).

[71] Kathryn M. Zurek Physics Reports 537, 91 (2014).

[72] S. Mukhopadhyay, D. Atta, K. Imam, D. N. Basu, C. Samanta, Eur. Phys. J. C, 77, 440 (2017).

[73] Eric Gourgoulhon arXiv:1003.5015v2 (2011).

[74] A. M. Lane, Nucl. Phys. 35, 676 (1962).

[75] G. R. Satchler, Int. series of monographs on Physics, Oxford University Press, Direct Nuclear reactions, 470 (1983).

[76] Gaurav Narain, Jürgen Schaffner-Bielich and Igor N. Mishustin, Phys. Rev. D 74, 063003 (2006). 